\title{
Active Disturbance Rejection Control based on Generalized Proportional Integral Observer to Control a Bipedal Robot with Five Degrees of Freedom
}

\author{
Jaime Arcos-Legarda ${ }^{1}$, John Cortes-Romero ${ }^{2}$ and A. Tovar ${ }^{3}$
}

\begin{abstract}
An Active Disturbance Rejection Control based on Generalized Proportional Integral observer (ADRC with GPI observer) was developed to control the gait of a bipedal robot with five degrees of freedom. The bipedal robot used is a passive point feet which produces an underactuated dynamic walking. A virtual holonomic constraint is imposed to generate online smooth trajectories which were used as references of the control system. The proposed control strategy is tested through numerical simulation on a task of forward walking with the robot exposed to external disturbances. The performance of ADRC with GPI observer strategy is compared with a feedback linearization with proportional-derivative control. A stability test consisting on analyzing the existence of limit cycles using the Poincaré's method revealed that asymptotically stable walking was achieved. The proposed control strategy effectively rejects the external disturbances and keeps the robot in a stable dynamic walking.
\end{abstract}

\section{INTRODUCTION}

This paper presents a control strategy aiming to overcome one of the main challenges in bipedal robot control systems, which is disturbance rejection. Although in the last few decades bipedal robots have had a remarkable development, control strategies to allow these devices to interact with the human environment are still in their infancy. Human environments have unpredictable sources of disturbances that affect the normal performance of a robot, reason for which methods to ensure stability are needed.

Several attempts have been made to solve the problems brought about by external disturbances and model uncertainties over bipedal robots. The first and most common approach is the use of strategies to adapt the walking pattern in order to change the trajectory references; its deployment gives the robot the ability to put the swing foot on a place where it finds balance easily [1]-[3].

A second approach is to improve the robustness of the control system that tracks the references [4]. Although the tracking control must be resistant to external disturbances, little development is observed in this area. Achieving robustness with the use of this control is highly important because of the incapability of tracking the references could result in the instability of the full robot. In consequence, in this case the quality of the strategy to generate references becomes

\footnotetext{
${ }^{1}$ Department of Mechanic and Mechatronic Engineering, Universidad Nacional de Colombia, Bogotá, Colombia. wjarcosl@unal . edu . co

${ }^{2}$ Department of Electric and Electronics Engineering, Universidad Nacional de Colombia, Bogotá, Colombia. jacortesr@unal .edu.co

${ }^{3}$ Mechanical Engineering, Indiana University-Purdue University Indianapolis Indianapolis, Indiana, USA. tovara@iupui . edu
}

no longer relevant. Most of the times this approach uses references that depend on the position of the robot instead on the time [5], which allows it to adapt its trajectories to the state of the gait.

Other strategies to detect the external disturbances use redundant systems of sensors and are based on simplified models as 1D Linear Inverted Pendulum dynamics model [6]. Those methods make estimations of the states and disturbances $[7,8]$. The use of simplified models has permitted the design of external forces observers using inertial sensors [9], but the results regarding stability are limited by the poor accuracy of the models. Arrays of sensors are used to homogenize the reaction forces on the feet; although this allows to reject external forces, it is constrained by the condition of planar contact of the feet with the ground [10].

This work presents an alternative control system for bipedal robots based on disturbances estimation and their active rejection. Thus, the main contribution of this work is to provide an effective control strategy to reject disturbances. External perturbations and model uncertainties are lumped in a single group of signals called total disturbances. Taking into account that the system has a multivariable and nonlinear model, the total disturbances were estimated with an extended observer approach, which assumes that the total disturbances are uniformly absolutely bounded away from zero and are smooth in all of its arguments [11]. Additionally it was considered that the total disturbances can be approximated by $m-1$ finite order polynomials. Based on this assumption observers with $m$ extended states were proposed as this was the approach used in the GPI control concept [12].

The outline of this paper is as follows: First, a model of a five degrees of freedom bipedal robot is presented in Section II. Then, the disturbances observer and the control strategy is proposed in Section III. After this, Section IV presents a reference trajectory design based on the robot's state. After that, global stability is tested by Poincaré's return map in Section V. Once established, simulations of the walking control were performed in Section VI. Finally, conclusions are draw in Section VII.

\section{ROBOT MODEL}

The robot's model was obtained by studying the multibody system formed by the bipedal robot that is shown in the Fig. 1. The bipedal walking is a periodic sequence of changes between single and double support. If it is assumed that the 
double support phase is instantaneous, then the single support phase would describe the longest phase of the walking [5, 13]. The Lagrange's differential equation can be used to find the model of the robot in the single support phase to get the Euler-Lagrange expressed as:

$$
D_{s}\left(q_{s}\right) \ddot{q}_{s}+C\left(q_{s}, \dot{q}_{s}\right) \dot{q}_{s}+G_{s}\left(q_{s}\right)=B_{s}\left(q_{s}\right) u+\delta\left(q_{s}, \dot{q}_{s}\right)+\zeta,
$$

where $q_{s}:=\left[\begin{array}{llll}q_{1} & q_{2} & \ldots & q_{5}\end{array}\right]^{\prime}$ is the generalized coordinates vector (The expression $[\bullet]^{\prime}$ represents the transpose), $D_{s}\left(q_{s}\right)$ is the inertial matrix, $C_{s}\left(q_{s}, \dot{q}_{s}\right) \dot{q}_{s}$ is a vector of centripetal and coriolis forces, $G_{s}\left(q_{s}\right)$ is a vector of forces associated to the gravity, $B_{s}\left(q_{s}\right) u$ is a vector of generalized forces, $\delta\left(q_{s}, \dot{q}_{s}\right)$ takes into account the uncertainties of the model and $\zeta$ is an unknown vector of the external disturbances. This model was acquired based on the assumption that the support leg is pinned to the ground with a revolute joint. Although, the

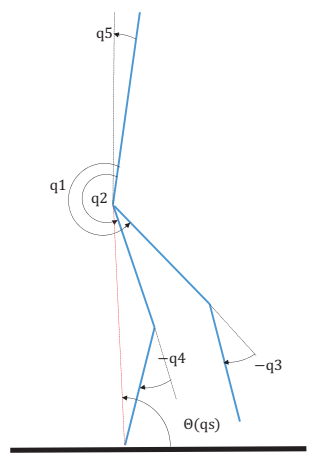

Fig. 1. Robot model

assumption of the instantaneous double support phase is true over few special conditions, it is not possible to avoid the impact that takes place in the change of support leg. That is why it is necessary to model the impact phase and to form a hybrid model with the continuous model of the single support phase and the discrete event in the foot switching at the moment of impact $[14,15]$. External forces affecting the system are a mandatory consideration when it comes to finding a model for the impact event. For this reason in this phase of the walking, the model was studied as an unpinned system and it was included a fix point over the body of the robot with its coordinates referenced to a fix frame, as it is shown below $p_{e}:=\left[\begin{array}{ll}p_{e}^{h} & p_{e}^{v}\end{array}\right]^{\prime}$, where $p_{e}^{h}$ refers to the horizontal coordinate of the point $p_{e}, p_{e}^{v}$ to the vertical one and $p_{e}$ was the position of the end of the support leg, this information enlarged the generalized coordinate vector, which took the following form $q_{e}:=\left[\begin{array}{ll}q_{s}^{\prime} & p_{e}^{\prime}\end{array}\right]^{\prime}$. With the new generalized coordinate vector clear, the dynamical model is described as below:

$$
D_{e}\left(q_{e}\right) \ddot{q}_{e}+C_{e}\left(q_{e}, \dot{q}_{e}\right) \dot{q}_{e}+G_{e}\left(q_{e}\right)=B_{e}\left(q_{e}\right) u+\delta F_{e x t},
$$

where $\delta F_{\text {ext }}$ represents impulsive forces caused by the ground reaction in the swing leg at the impact time. Based on the concept of momentum conservation, the following equation was obtained:

$$
D_{e}\left(q_{e}^{+}\right) \dot{q}_{e}^{+}-D_{e}\left(q_{e}^{-}\right) \dot{q}_{e}^{-}=F_{e x t},
$$

where $\left(q_{e}^{-}, \dot{q}_{e}^{-}\right)$and $\left(q_{e}^{+}, \dot{q}_{e}^{+}\right)$represent the position and the velocity just before and after the impact respectively; $F_{\text {ext }}$ is a forces vector which represents the effect of the ground reaction on each joint. Define $F_{2}:=\left[\begin{array}{ll}F_{2}^{T} & F_{2}^{N}\end{array}\right]^{\prime}$ as the reaction force in the swing leg, where $F_{2}^{T}$ refers to the tangential reaction and $F_{2}^{N}$ to the normal one. Following the principle of virtual work, it yields:

$$
F_{\text {ext }}=E_{2}\left(q_{e}^{-}\right)^{\prime} F_{2}
$$

where $E_{2}:=\frac{\partial p_{2}}{\partial q_{e}}$ and $p_{2}$ is the position of the swing leg end. Replacing the Eq. (4) into (3) it produces:

$$
D_{e}\left(q_{e}^{-}\right) \dot{q}_{e}^{-}=D_{e}\left(q_{e}^{+}\right) \dot{q}_{e}^{+}+E_{2}\left(q_{e}^{-}\right)^{\prime} F_{2}
$$

If the hypothesis that the impact is totally plastic is accepted, it would be true that there exist no rebounds or slips in the stance foot, which permits us to conclude that the position of the mechanism would not change after the impact, it is $q_{e}^{-} \equiv q_{e}^{+}$, and the velocity of the end leg would be equal to zero as shown below:

$$
\begin{array}{r}
\frac{d p_{2}}{d t}=\frac{\partial p_{2}\left(q_{e}^{-}\right)}{\partial q_{e}} \dot{q}_{e}^{+}, \\
E_{2}\left(q_{e}^{-}\right) \dot{q}_{e}^{+}=0 .
\end{array}
$$

The Eq. (5) and (6) are collected in a matrix form to obtain

$$
\left[\begin{array}{c}
\dot{q}_{e}^{+} \\
F_{2}
\end{array}\right]=\left[\begin{array}{cc}
D_{e}\left(q_{e}^{+}\right) & -E_{2}\left(q_{e}^{-}\right)^{\prime} \\
E_{2}\left(q_{e}^{-}\right) & 0_{2 \times 2}
\end{array}\right]^{-1}\left[\begin{array}{c}
D_{e}\left(q_{e}^{-}\right) \dot{q}_{e}^{-} \\
0_{2 \times 1}
\end{array}\right],
$$

which can be summarized in

$$
\begin{aligned}
\dot{q}_{e}^{+} & =\Delta\left(q_{e}^{-}, \dot{q}_{e}^{-}\right), \\
F_{2} & =\Sigma\left(q_{e}^{-}, \dot{q}_{e}^{-}\right),
\end{aligned}
$$

Finally, the hybrid system is described in the Eq. (1) and (8) as:

$$
\Sigma:\left\{\begin{array}{cc}
\ddot{q}_{s}=f\left(q_{s}, \dot{q}_{s}\right)+D_{s}\left(q_{s}\right)^{-1} B_{s}\left(q_{s}\right) u & p_{2}^{v} \neq 0 \\
\dot{q}_{e}^{+}=\Delta\left(q_{e}^{-}, \dot{q}_{e}^{-}\right) & p_{2}^{v}=0
\end{array}\right.
$$

where

$f\left(q_{s}, \dot{q}_{s}\right)=D_{s}\left(q_{s}\right)^{-1}\left[-C\left(q_{s}, \dot{q}_{s}\right) \dot{q}_{s}-G_{s}\left(q_{s}\right)+\delta\left(q_{s}, \dot{q}_{s}\right)+\zeta\right]$.

A more detailed description of the model can be found in $[5,13]$.

\section{CONTROL DESIGN}

The problem of bipedal robot's control is divided into two main control loops, as it is displayed in Fig. 2. The external loop has the task of generating the walking pattern, which can either be time function or function of the robot state, this problem will be studied in the following section. The internal control loop has the task of tracking references generated by the external loop. In this paper a control strategy for the internal loop intended to keep the tracking error close to zero, reject the external disturbances and overcome the model uncertainties was developed.

Now, let us analyze the features of the problem. First, it is clear that this system is a nonlinear and multi-input multioutput system. This problem has an additional characteristic that makes it a non-conventional problem, because it is an 


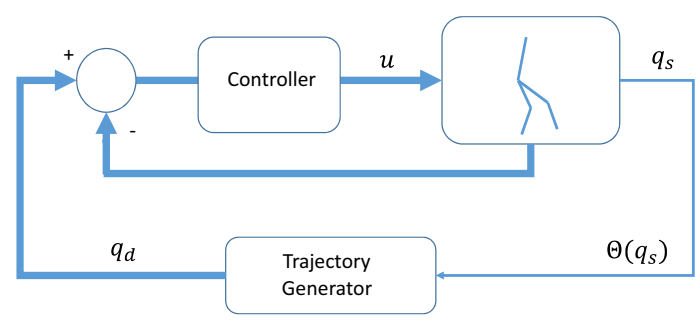

Fig. 2. control problem

underactuated system. Although the model of the robot in Eq. (1) shows that there are five degrees of freedom, the robot has four actuators only, one at each knee and one at each side of the hip. To overcome the difficulties to control design brought about by this underactuated feature of the system the Eq. (1) is simplified below:

$$
D_{s}\left(q_{s}\right) \ddot{q}_{s}+\Omega\left(q_{s}, \dot{q}_{s}\right)=B_{s}\left(q_{s}\right) u+\tilde{\xi}\left(q_{s}, \dot{q}_{s}\right),
$$

where:

$\Omega\left(q_{s}, \dot{q}_{s}\right):=C\left(q_{s}, \dot{q}_{s}\right) \dot{q}_{s}+G_{s}\left(q_{s}\right)$, $\tilde{\xi}\left(q_{s}, \dot{q}_{s}\right):=\delta\left(q_{s}, \dot{q}_{s}\right)+\zeta$.

The Eq. (11) is fragmented to separate the actuated part of the system out of underactuated part. Now, let us fragment the position vector and the output matrix as

$$
q_{s}:=\left[\begin{array}{c}
q_{b_{(N-1 \times 1)}} \\
q_{\left(N_{1 \times 1}\right)}
\end{array}\right], \quad B_{s}\left(q_{s}\right):=\left[\begin{array}{c}
B_{1}\left(q_{s}\right)_{(N-1 \times p)} \\
\left.0_{(1 \times p)}\right]
\end{array}\right],
$$

where $p$ is the number of actuators. Once the new structure of the variables was established, the model was fragmented as:

$$
\begin{gathered}
{\left[\begin{array}{ll}
D_{11}\left(q_{s}\right) & D_{12}\left(q_{s}\right) \\
D_{21}\left(q_{s}\right) & D_{22}\left(q_{s}\right)
\end{array}\right]\left[\begin{array}{c}
\ddot{q}_{b} \\
\ddot{q}_{N}
\end{array}\right]+\left[\begin{array}{l}
\Omega_{1}\left(q_{s}, \dot{q}_{s}\right) \\
\Omega_{2}\left(q_{s}, \dot{q}_{s}\right)
\end{array}\right]=\ldots} \\
\cdots=\left[\begin{array}{c}
B_{1}\left(q_{s}\right) \\
0
\end{array}\right] u+\left[\begin{array}{l}
\tilde{\xi}_{1}\left(q_{s}, \dot{q}_{s}\right) \\
\widetilde{\xi}_{2}\left(q_{s}, \dot{q}_{s}\right)
\end{array}\right]
\end{gathered}
$$

The fragmented model representation is used to express the model as a function of the actuated variables, which is

$$
\begin{array}{r}
{\left[D_{11}\left(q_{s}\right)-D_{12}\left(q_{s}\right) D_{22}^{-1}\left(q_{s}\right) D_{21}\left(q_{s}\right)\right] \ddot{q}_{b}+\Omega_{1}\left(q_{s}, \dot{q}_{s}\right)+\ldots} \\
\ldots+D_{12}\left(q_{s}\right) D_{22}^{-1}\left(q_{s}\right)\left[\tilde{\xi}_{2}\left(q_{s}, \dot{q}_{s}\right)-\Omega_{2}\left(q_{s}, \dot{q}_{s}\right)\right]=\ldots \\
\ldots=B_{1}\left(q_{s}\right) u+\tilde{\xi}_{1}\left(q_{s}, \dot{q}_{s}\right) \\
\cdots
\end{array}
$$

then, it is simplified to obtain

$$
\ddot{q}_{b}=\kappa\left(q_{s}\right) u+\xi
$$

where

$$
\begin{array}{r}
\xi=\left[D_{11}\left(q_{s}\right)-D_{12}\left(q_{s}\right) D_{22}^{-1}\left(q_{s}\right) D_{21}\left(q_{s}\right)\right]^{-1} * \ldots \\
\ldots *\left\{-D_{12}\left(q_{s}\right) D_{22}\left(q_{s}\right)^{-1}\left[\tilde{\xi}_{2}\left(q_{s}, \dot{q}_{s}\right)-\Omega_{2}\left(q_{s}, \dot{q}_{s}\right)\right]+\ldots\right. \\
\left.\ldots+\tilde{\xi}_{1}\left(q_{s}, \dot{q}_{s}\right)-\Omega_{1}\left(q_{s}, \dot{q}_{s}\right)\right\},
\end{array}
$$

and

$$
\kappa\left(q_{s}\right)=\left[D_{11}\left(q_{s}\right)-D_{12}\left(q_{s}\right) D_{22}^{-1}\left(q_{s}\right) D_{21}\left(q_{s}\right)\right]^{-1} B_{1}\left(q_{s}\right) .
$$

\section{A. Design of Generalized Proportional Integral Observer}

Thanks to the ADRC approach, the MIMO (multi-input and multi-output) (1) latter simplified as is shown in Eq. (13) represents a system which can be treated as decoupled system, therefore, the control strategy is reduced to a problem of designing a group of SISO controllers (single-input and single-output) with disturbance rejection [16]. In order to design an observer to do estimations of the disturbances, the simplified system is represented in a extended state space form.

It is supposed that $\xi_{i}$ can be approximated by a polynomial with order $m-1$, where $m>1$, thereby $\frac{d^{m} \xi_{i}}{d t^{m}} \approx 0$. This is the main difference between the classical ADRC approach, that uses only one extended state and the ADRC with GPI observer which uses more than one. This approach permits to avoid the depoyment of high gains in the observer which is an advantage in the process of implementation. It is defined $x_{1, i}:=q_{b_{i}}, x_{2, i}:=\dot{q}_{b_{i}}$, and $x_{3, i}:=\xi_{i}$, for all the values that takes $i$, then the system takes the following form:

$$
\left.\begin{array}{l}
\dot{x}_{1, i}=x_{2, i}, \\
\dot{x}_{2, i}=x_{3, i}+\kappa_{i} u_{i}, \\
\dot{x}_{3, i}=x_{4, i}, \\
\vdots \\
\dot{x}_{m+1, i}=x_{m+2, i}, \\
\dot{x}_{m+2, i}=\frac{d^{m} \xi_{i}}{d t^{m}}
\end{array}\right\} \forall i=1, \ldots, N-1 .
$$

With the model in state space established, the GPI observer is proposed,

$$
\left.\begin{array}{l}
\dot{\hat{x}}_{1, i}=\hat{x}_{2, i}+l_{m+1, i}\left(x_{1, i}-\hat{x}_{1, i}\right), \\
\dot{\hat{x}}_{2, i}=\hat{x}_{3, i}+l_{m, i}\left(x_{1, i}-\hat{x}_{1, i}\right)+\kappa_{i} u_{i}, \\
\dot{\hat{x}}_{3, i}=\hat{x}_{4, i}+l_{m-1, i}\left(x_{1, i}-\hat{x}_{1, i}\right), \\
\vdots \\
\dot{\hat{x}}_{m+1, i}=\hat{x}_{m+2, i}+l_{1, i}\left(x_{1, i}-\hat{x}_{1, i}\right), \\
\dot{\hat{x}}_{m+2, i}=l_{0, i}\left(x_{1, i}-\hat{x}_{1, i}\right) .
\end{array}\right\} \forall i=1, \ldots, N-1 .
$$

Subtracting the observer Eq. (17) from the system state space representation (16) and defining the estimation error as:

$$
\left[\begin{array}{lll}
\hat{e}_{1, i} & \ldots & \hat{e}_{N-1, i}
\end{array}\right]^{\prime}=\left[\begin{array}{lll}
\left(x_{1, i}-\hat{x}_{1, i}\right) & \ldots & \left(x_{N-1, i}-\hat{x}_{N-1, i}\right)
\end{array}\right]^{\prime}
$$

then, the estimation error dynamics can be described by

$$
\left.\begin{array}{l}
\dot{\hat{e}}_{1, i}=\hat{e}_{2, i}-l_{m+1, i} \hat{e}_{1, i}, \\
\dot{\hat{e}}_{2, i}=\hat{e}_{3, i}-l_{m, i} \hat{e}_{1, i}+\kappa_{i} u_{i}, \\
\dot{\hat{e}}_{3, i}=\hat{e}_{4, i}-l_{m-1, i} \hat{e}_{1, i}, \\
\vdots \\
\dot{\hat{e}}_{m+1, i}=\hat{e}_{m+2, i}-l_{1, i} \hat{e}_{1, i}, \\
\dot{\hat{e}}_{m+2, i}=\frac{d^{m} \xi_{i}}{d t^{m}}-l_{0, i} e_{1, i} .
\end{array}\right\} \forall i=1, \ldots, N-1,
$$

or in a matrix form

$$
\left.\begin{array}{l}
\hat{\mathbf{e}}_{i}=\hat{\mathbf{A}}_{i} \hat{\mathbf{e}}_{i}+\hat{\mathbf{B}} \frac{d^{m} \xi_{i}}{d t^{m}}, \\
\mathbf{y}_{i}=\hat{\mathbf{C}} \hat{\mathbf{e}}_{i} .
\end{array}\right\} \forall i=1, \ldots, N-1,
$$


where

$$
\begin{gathered}
\hat{\mathbf{A}}_{i}=\left[\begin{array}{cccccc}
-l_{m+1, i} & 1 & 0 & \cdots & 0 & 0 \\
-l_{m, i} & 0 & 1 & \ldots & 0 & 0 \\
-l_{m-1, i} & 0 & 0 & \cdots & 0 & 0 \\
\vdots & \vdots & \vdots & \ddots & \ddots & \vdots \\
-l_{1, i} & 0 & 0 & \cdots & 0 & 1 \\
-l_{0, i} & 0 & 0 & \cdots & 0 & 0
\end{array}\right] ; \hat{\mathbf{B}}=\left[\begin{array}{c}
0 \\
0 \\
0 \\
\vdots \\
0 \\
1
\end{array}\right] ; \\
\hat{\mathbf{C}}=\left[\begin{array}{llllll}
1 & 0 & 0 & \cdots & 0 & 0
\end{array}\right] .
\end{gathered}
$$

The matrices $\hat{\mathbf{A}}_{i}$ must be appropriately selected in order to make the system described by the Eq. (19) be Hurwitz so it will achieve an asymptotically stable observer. A way to assign the values of $\hat{\mathbf{A}}_{i}$ can be found in [17].

\section{B. Control law proposition}

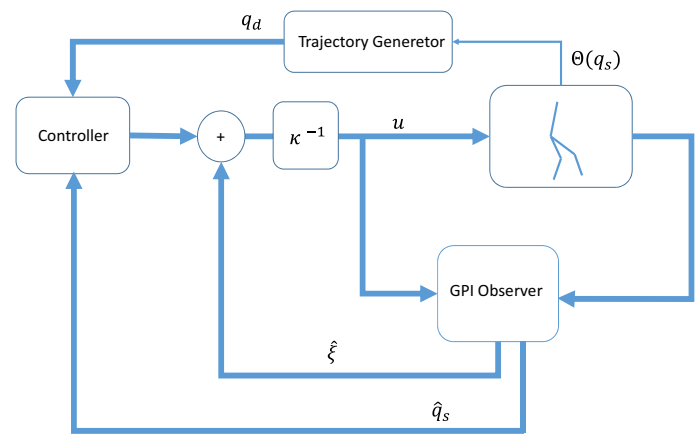

Fig. 3. control ADRC

Once the observer has been designed, it is proposed a control structure as is shown in the Fig. 3. This structure states a control law for tracking references. In the control law the disturbance estimation vector, $\hat{\xi}:=\hat{x}_{3}=$ $\left[\begin{array}{llll}\hat{x}_{3,1} & \hat{x}_{3,2} & \ldots & \hat{x}_{3, N-1}\end{array}\right]^{\prime}$, is used to reject its effects in the system. Furthermore, the estimation of position vector, $\hat{q}_{b}:=\hat{x}_{1}=\left[\begin{array}{llll}\hat{x}_{1,1} & \hat{x}_{1,2} & \ldots & \hat{x}_{1, N-1}\end{array}\right]^{\prime}$, and velocity vector, $\hat{\dot{q}}_{b}:=\hat{x}_{2}=\left[\begin{array}{llll}\hat{x}_{2,1} & \hat{x}_{2,2} & \ldots & \hat{x}_{2, N-1}\end{array}\right]^{\prime}$, are used to build a proportional-derivative control action, as is show below:

$$
u=\kappa^{-1}\left(\ddot{q}_{d}-K_{d}\left(\hat{x}_{2}-\dot{q}_{d}\right)-K_{p}\left(\hat{x}_{1}-q_{d}\right)-\hat{\xi}\right),
$$

where $q_{d}$ is the reference vector and $K_{p}$ and $K_{d}$ are diagonal matrices. This control law has the form of a proportionalderivative (PD) control assisted by GPI observer, whose results were compared with the ones obtained from a classical feedback linearization with PD control, without assistant observer.

Replacing the control law (20) into the simplified system (13), it gives the following equation:

$$
\left(\ddot{q}_{b}-\ddot{q}_{d}\right)+K_{d}\left(\hat{x}_{2}-\dot{q}_{d}\right)+K_{p}\left(\hat{x}_{1}-q_{d}\right)=\xi-\hat{\xi},
$$

which is a decoupled equation system. Although the Eq. (21) shows the close loop dynamics, it does not allow us draw any conclusion about the tracking error. In order to get a relation of the close loop dynamics as a function of the tracking error, let us define the error position estimation as $\hat{e}_{1}=\left[\begin{array}{llll}\hat{e}_{1,1} & \hat{e}_{1,2} & \ldots & \hat{e}_{1, N-1}^{\prime}\end{array}\right.$ and taking into account that $\hat{x}_{1}=x_{1}-\hat{e}_{1}, x_{1}=q_{b}$ and $\hat{x}_{2}=\dot{\hat{x}}_{1}-l_{m+1}\left(x_{1}-\hat{x}\right)$, then the close loop system takes the following form:

$$
\begin{array}{r}
\left(\ddot{q}_{b}-\ddot{q}_{d}\right)+K_{d}\left(\dot{q}_{b}-\dot{q}_{d}\right)+K_{p}\left(q_{b}-q_{d}\right)=\ldots \\
\cdots=K_{d} \dot{\hat{e}}_{1}+\left(K_{p}+l_{m+1}\right) \hat{e}_{1}+(\xi-\hat{\xi}) .
\end{array}
$$

Applying the Laplace transform to the Eq. (22) and defining the tracking error as $e_{q_{i}}=\left(q_{b_{i}}-q_{d_{i}}\right), \forall i=1, \ldots, N-$ 1 ; then the tracking error dynamics is described by the following transfer function:

$$
\begin{aligned}
e_{q_{i}}(s)= & \frac{K_{d_{(i, i)} s+\left(K_{p_{(i, i)}}+l_{m+1, i}\right)}}{s^{2}+K_{d_{(i, i)}} s+K_{p_{(i, i)}}} \hat{e}_{1, i}(s)+\ldots \\
& \cdots+\frac{\left(\xi_{i}(s)-\hat{\xi}_{i}(s)\right)}{s^{2}+K_{d_{(i, i)}} s+K_{p_{(i, i)}}}, \forall i=1, \ldots, N-1,
\end{aligned}
$$

which shows that the tracking error tend asymptotically to zero if:

1) the estimation errors tend to zero,

2) and, $K_{d_{i}}, K_{p_{i}}$ are chosen appropriately.

\section{TRAJECTORY GenERATION}

The generation of trajectories or references for the joints is a task that can be solved by an external control loop. One of the most common approaches in the references generation is to do it off-line and use them as function of the time. Given that this approach has not the flexibility needed to reject disturbances. In this work uses a methodology for generation of trajectories as function of the state of the robot. This methodology is better know as "virtual holonomic constraint" and is employed to ensure that the references of the inner control loop will be the correct ones to get a stable periodic gait [15].

Although the aim of this paper is not to reject the disturbances through a modification of the trajectories, the way to generate a gait pattern presented here permits to analyze the performance of the tracking controls independently from those. Before starting with the process of generation of trajectories, few parameters were established taking as inspiration the human-like walking, thus, the trajectory for the hip is taken as a sine function and the step length is a fraction of the human step length. Additionally to the mentioned above, conditions to achieve smooth trajectories are included. The trajectory of each joint is described by a high order polynomial regression, which is function of the absolute angle $\Theta(q s)$ shown in the Fig. 1. Those polynomials produce the vector of references $q_{d}$.

\section{Stability Test}

The bipedal gait is a periodic sequence of states. This periodicity makes the stability analysis a special challenge because the asymptotic stability can not be established by the classical sense of Lyapunov for equilibrium points. Thus, the method of Poincaré's section is used identify periodic behavior in the dynamic of the biped. The Poincaré's method searches for the presence of a periodic orbit in the evolution of the states; it is done through the sampling of the states in a time when a condition is satisfied. Such a condition is called the Poincaré's section. Poincaré's section could either 
be a function of few robot states or can be determined by external events. This is the case of the bipedal gait where the Poincaré's section for the bipedal gait is determined by the switching foot event, which depends of the ground level.

The sampling done in the Poincaré's section is used to build a function that takes values just before the impact time and it is defined as

$$
x^{-}(k+1)=P\left(x^{-}(k)\right)
$$

where $x^{-}(k)$ is the $k^{\text {th }}$ sample of the state vector just before the impact, $x^{-}(k+1)$ is the state vector just before the next impact and $P\left(x^{-}(k)\right)$ is a function called the Poincaré return map. The stability test is summarized in the tasks of evaluating the existence of an equilibrium point $x^{-*}$ such that

$$
x^{-}(k+1)=P\left(x^{-*}(k)\right)=x^{-*}
$$

as well as in tasks of demonstrating the discrete system stability of (24) in the classical sense of Lyapunov for the equilibrium point $x^{-*}$.

Given the complexity of determining the Poincaré return map, $P\left(x^{-}(k)\right)$, it is often calculated through numerical simulations of the robot walking. The states of the robot in each switching foot are saved in a vector

$$
\chi(k)=\left[\begin{array}{c}
x^{-}(k-(\rho-1))-x^{-^{*}} \\
x^{-}(k-(\rho-2))-x^{-^{*}} \\
\vdots \\
x^{-}(k)-x^{-^{*}}
\end{array}\right],
$$

where $\rho$ represents the number of switching feet executed in the simulation. A linear regression is performed between the $\chi(k)$ and $\chi(k-1)$ values. The result of the regression gives a linear approximation of (24) with the following form

$$
\left(x^{-}(k+1)-x^{-^{*}}\right)=\Phi *\left(x^{-}(k)-x^{-^{*}}\right)
$$

where the eigenvalues of $\Phi$ must have a magnitude lower than one to guarantee the asymptotic stability of the walking.

\section{SIMULATIONS AND RESULTS}

The control strategy, the extended observer and the trajectories generator proposed at the above sections were evaluated through simulation of the bipedal robot in the task of forward walking over a flat ground. Before evaluating the systems, parameters like the order of the approximation of the total disturbance, the observer and the controller constants need to be established. First, the order of total disturbance, $\xi$, which is approximated by $m=7$. Second, the observer constants, that were calculated to define the dynamic of the observer is described by the eigenvalues of the matrix $\hat{\mathbf{A}}$, Shown in the Table I. Finally, the controller constants were selected as shown in the Table II.

The simulations of the walking robot controlled by ADRC with GPI observer was compared with a classical control strategy like feedback linearization with proportionalderivative action, called from now on NPD (Nonlinear P-D). The control law for the NPD proposed takes the form

$$
u=\kappa^{-1}\left(\ddot{q}_{d}-K_{d}\left(\hat{x}_{2}-\dot{q}_{d}\right)-K_{p}\left(\hat{x}_{1}-q_{d}\right)-\xi\right) .
$$

where $\xi$ is taken from the model shown in the Eq. (14), but with $\tilde{\xi}_{1}\left(q_{s}, \dot{q}_{s}\right)=0$ and $\tilde{\xi}_{2}\left(q_{s}, \dot{q}_{s}\right)=0$ [13].

TABLE I

OBSERVER EIGENVALUES

\begin{tabular}{|l|lllll|}
\hline$i$ & \multicolumn{5}{|c|}{ Eigenvalues } \\
\hline$\forall i$ & -180, & -324, & -583, & -1050, & -1890, \\
& -3401, & -6122, & -11.020, & -19.836, & \\
\hline
\end{tabular}

TABLE II

CONTROLLER CONSTANTS

\begin{tabular}{|l|c|c|}
\hline$i$ & $K_{p_{(i, i)}}$ & $K_{d_{(i, i)}}$ \\
\hline$\forall i$ & 5000 & 50 \\
\hline
\end{tabular}

In order to get a comparable dynamic between the control strategies, the constants used by NPD controller do not differ from the ones used in the Table II. The evaluation below intends to contrast the performance of the two strategies and therefore, a test of each one of them undergoes a test of 12.5 steps (25 swithing foot) done for each one. These simulations evaluate the capacity to reject external disturbances, which were emulated through torques applied to the joints of the system and injected in the $10^{\text {th }}$ steep.

Figure 4 shows the tracking references of the control NPD, the control signals and its response to the external disturbance. Even though the system has the capacity to keep the stability, the perturbation affects the controller's performance. On the other hand, Figure 5 shows the performance of the controller ADRC with GPI observer, which has an effective disturbance rejection without deviation of the tracking reference.

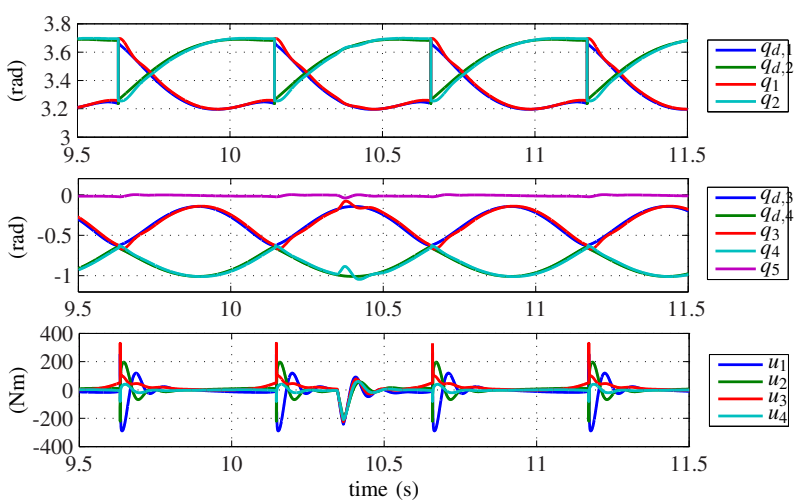

Fig. 4. Performance NPD

The progression of $\Theta\left(q_{s}\right)$ vs $d / d t \Theta\left(q_{s}\right)$ of both tests, shown in Figure 6, depicts the feature of a cycle limit of the walking. It also shows that the ADRC control has not been affected by the external disturbances and that the stability of the cycle limit is not under risk. On the other hand, the control NPD does not have the ability to guarantee the stability if the system is exposed to external disturbances in a critical phase of the gait.

The stability of the bipedal robot in the simulation of ADRC with GPI observer was tested through the Poincaré's 

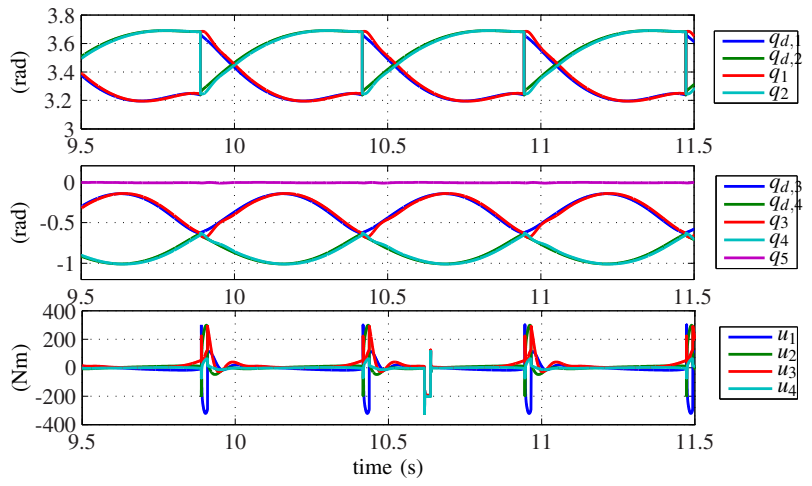

Fig. 5. Performance ADRC with GPI observer

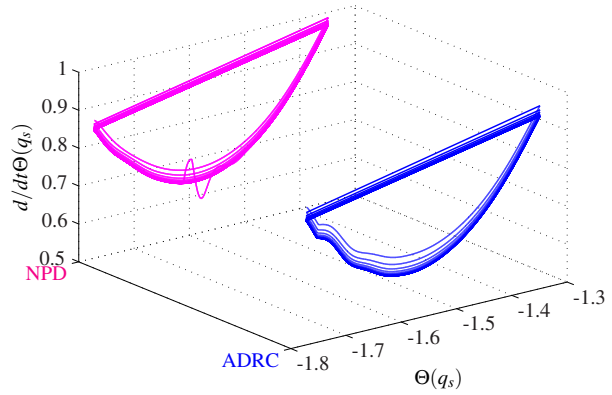

Fig. 6. Limit cycles

method. First was calculated the equilibrium point in the Poincaré map, which is equal to:

$x^{-^{*}}=\left[\begin{array}{ccccc}3.247 & 3.672 & -0.622 & -0.644 & -0.0104 \cdots \\ \cdots-0.298 & 0.340 & -1.578 & 1.370 & -0.170\end{array}\right]$.

Second the values of the sampling states in the Poincaré section were used to find $\Phi$ with a linear regression. Finally the eigenvalues of $\Phi$ were found and their magnitudes were calculated to obtain $|\lambda(\Phi)|$. Given that all the eigenvalues, $\lambda(\Phi)$, were inside a unitary circle, then it can be concluded that the control system is asymptotically stable.

$$
|\lambda(\Phi)|=\left[\begin{array}{ccccc}
0.640 & 0.640 & 0.844 & 0.844 & 0.237 \cdots \\
\cdots 0.237 & 0.138 & 0.354 & 0.354 & 0.6609
\end{array}\right] .
$$

\section{CONCLUSIONS}

An Active Disturbance Rejection Control with Generalized Proportional Integral observer approach was proposed to control the tracking reference of an underactuated bipedal robot. This device achieved a periodically asymptotically stable walking. A performance comparison between the proposed control and a classical controller showed the benefits of the ADRC with GPI in tracking references and disturbances rejection.

An effective control strategy to reject external disturbances in the gait of bipedal robots was developed through the use of active disturbance rejection control based on generalized proportional integral observer. Simulations on the control strategy proposed evidenced its capability to reject external disturbances as well as its capability to achieve asymptotically stable walking.
The nominal walking pattern projected was a successful virtual holonomic constraint to achieve a stable gait. Although the trajectories generation method was successful to keep the robot equilibrium, it did not provide an optimal trajectory reference to minimize energy consumption and neither gave a smooth enough trajectory in the impact.

\section{REFERENCES}

[1] S. Czarnetzki, S. Kerner, and O. Urbann, "Observer-based dynamic walking control for biped robots," Robotics and Autonomous Systems, vol. 57 , no. 8, pp. 839-845, 2009.

[2] T. Koolen, T. De Boer, J. Rebula, A. Goswami, and J. Pratt, "Capturability-based analysis and control of legged locomotion, part 1: Theory and application to three simple gait models," The International Journal of Robotics Research, vol. 31, no. 9, pp. 1094-1113, 2012.

[3] J. Pratt, T. Koolen, T. De Boer, J. Rebula, S. Cotton, J. Carff, M. Johnson, and P. Neuhaus, "Capturability-based analysis and control of legged locomotion, part 2: Application to m2v2, a lower body humanoid," The International Journal of Robotics Research, p. $0278364912452762,2012$.

[4] J. Arcos-Legarda, A. Tovar, J. Cortés, H. Díaz, and L. Sarmiento, "Multivariable gpi control of a gait exoskeleton for people with walking disabilities," in II International Congress of Engineering Mechatronics and Automation (CIIMA), 2013.

[5] S. Tzafestas, M. Raibert, and C. Tzafestas, "Robust sliding-mode control applied to a 5-link biped robot," Journal of Intelligent and Robotic Systems, vol. 15, no. 1, pp. 67-133, 1996.

[6] S. Kajita and K. Tan, "Study of dynamic biped locomotion on rugged terrain-derivation and application of the linear inverted pendulum mode," in Robotics and Automation, 1991. Proceedings., 1991 IEEE International Conference on, pp. 1405-1411, IEEE, 1991.

[7] B. J. Stephens, "State estimation for force-controlled humanoid balance using simple models in the presence of modeling error," in Robotics and Automation (ICRA), 2011 IEEE International Conference on, pp. 3994-3999, IEEE, 2011.

[8] I. Hashlamon and K. Erbatur, "Center of mass states and disturbance estimation for a walking biped," in Mechatronics (ICM), 2013 IEEE International Conference on, pp. 248-253, IEEE, 2013.

[9] K. Kaneko, F. Kanehiro, M. Morisawa, E. Yoshida, and J.-P. Laumond, "Disturbance observer that estimates external force acting on humanoid robots," in Advanced Motion Control (AMC), 2012 12th IEEE International Workshop on, pp. 1-6, IEEE, 2012.

[10] C. Ott, M. Roa, G. Hirzinger, et al., "Posture and balance control for biped robots based on contact force optimization," in Humanoid Robots (Humanoids), 2011 11th IEEE-RAS International Conference on, pp. 26-33, IEEE, 2011.

[11] R. Madoński and P. Herman, "Survey on methods of increasing the efficiency of extended state disturbance observers," ISA transactions, 2015.

[12] J. Cortés-Romero, G. A. Ramos, and H. Coral-Enriquez, "Generalized proportional integral control for periodic signals under active disturbance rejection approach," ISA transactions, vol. 53, no. 6, pp. 19011909, 2014

[13] E. R. Westervelt, J. W. Grizzle, C. Chevallereau, J. H. Choi, and B. Morris, Feedback control of dynamic bipedal robot locomotion, vol. 28. CRC press, 2007.

[14] J. W. Grizzle, G. Abba, and F. Plestan, "Asymptotically stable walking for biped robots: Analysis via systems with impulse effects," Automatic Control, IEEE Transactions on, vol. 46, no. 1, pp. 51-64, 2001.

[15] E. R. Westervelt, J. W. Grizzle, and D. E. Koditschek, "Hybrid zero dynamics of planar biped walkers," Automatic Control, IEEE Transactions on, vol. 48, no. 1, pp. 42-56, 2003.

[16] Q. Zheng, Z. Chen, and Z. Gao, "A practical approach to disturbance decoupling control," Control Engineering Practice, vol. 17, no. 9, pp. 1016-1025, 2009.

[17] Y. Huang and W. Xue, "Active disturbance rejection control: methodology and theoretical analysis," ISA transactions, vol. 53, no. 4, pp. 963976, 2014. 